\title{
Sommaire de la Déclaration du Comité consultatif national de l'immunisation sur la vaccination antigrippale pour la saison 2019-2020
}

\author{
L Zhao', K Young ${ }^{1}$, I Gemmill ${ }^{2,3}$ au nom du Comité consultatif national sur l'immunisation (CCNI)*
}

\section{Résumé}

Contexte : L'utilisation de nombreux vaccins antigrippaux différents est autorisée au Canada et de nouvelles données probantes sur la grippe et les vaccins antigrippaux font constamment leur apparition. Le Comité consultatif national de l'immunisation (CCNI) présente ses recommandations annuelles concernant l'utilisation des vaccins antigrippaux saisonniers à l'Agence de la santé publique du Canada (ASPC).

Objectif : Résumer les recommandations du CCNI concernant l'utilisation des vaccins antigrippaux pour la saison 2019-2020, y compris les conclusions des analyses de données probantes sur 1) un nouveau vaccin quadrivalent antigrippal inactivé à virion fragmenté et 2) l'efficacité et l'immunogénicité comparatives du vaccin antigrippal inactivé sous-unitaire et du vaccin antigrippal inactivé à virion fragmenté chez les adultes de 65 ans et plus.

Méthodologie : Pour ces deux sujets, le Groupe de travail sur l'influenza du CCNI a mis au point une stratégie de recherche prédéfinie pour identifier toutes les études admissibles, évaluer leur qualité, résumer et analyser les résultats et, conformément au processus fondé sur des données probantes du CCNI, proposer des recommandations et déterminer la cote de qualité des données probantes qui les appuient. À la lumière de la preuve, les recommandations ont ensuite été étudiées et approuvées par le CCNI.

Résultats : Le CCNI a conclu que le nouveau vaccin antigrippal quadrivalent inactivé à virion fragmenté a un profil d'innocuité et d'immunogénicité comparable à celui des vaccins antigrippaux quadrivalents inactivés déjà autorisés pour les adultes et les enfants de cinq ans et plus (Données probantes de catégorie B). Le CCNI recommande que ce nouveau vaccin puisse compter parmi les vaccins antigrippaux quadrivalents inactivés offerts aux adultes et aux enfants de cinq ans et plus (Recommandation discrétionnaire du CCNI). Le CCNI a cependant conclu que les données probantes ne sont pas suffisantes (Données probantes de catégorie I) pour appuyer des recommandations précises sur l'utilisation différentielle des vaccins antigrippaux inactivés sous-unitaire et à virion fragmenté chez les adultes de 65 ans et plus.

Conclusion : Le CCNI continue de recommander le vaccin antigrippal pour toute personne de six mois et plus qui ne présente aucune contre-indication à l'administration du vaccin, en accordant une importance particulière aux groupes pour lesquels le vaccin antigrippal est particulièrement recommandé. Cela inclut les personnes présentant un risque élevé de complications ou d'hospitalisation liées à la grippe, les personnes pouvant transmettre la grippe aux personnes à risque élevé, les personnes qui fournissent des services communautaires essentiels et les personnes en contact direct avec de la volaille infectée par le virus de la grippe aviaire durant les activités d'abattage.
Citation proposée : Zhao L, Young K, Gemmill I au nom du Comité consultatif national de l'immunisation (CCNI) Sommaire de la Déclaration du CCNI sur la vaccination antigrippale pour la saison 2019-2020. Relevé des maladies transmissibles au Canada 2019;45(6):165-71. https://doi.org/10.14745/ccdr.v45i06a01f

Mots clés : Comité consultatif national de l'immunisation, CCNI, influenza, grippe, vaccin antigrippal, conseils
Cette oeuvre est mise à la disposition selon les termes de la licence internationale Creative Commons Attribution 4.0

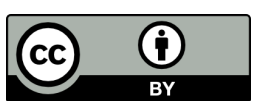

Affiliations

${ }^{1}$ Le Centre de l'immunisation et des maladies respiratoires infectieuses (CIMRI), Agence de la santé publique du Canada, Ottawa (Ontario)

2 Président du Groupe de travail sur l'influenza du CCNI

3 Université Queen's, Kingston (Ontario)

${ }^{\star}$ Correspondance : phac.naci-ccni.aspc@canada.ca 


\section{Introduction}

La grippe, avec la pneumonie, se range parmi les 10 principales causes de décès au Canada (1). Bien que le fardeau de la grippe puisse varier d'une année à l'autre, on estime qu'il y a en moyenne environ 12200 hospitalisations (2) et 3500 décès (3) liés à la grippe par année.

Le Comité consultatif national de l'immunisation (CCNI) présente des recommandations annuelles concernant I'utilisation des vaccins antigrippaux saisonniers à l'Agence de la santé publique du Canada (ASPC). Pour la saison grippale 2019-2020, le CCNI a mis à jour les abréviations utilisées pour les divers types de vaccins antigrippaux disponibles au Canada. Le CCNI a aussi examiné les données probantes de deux revues de la littérature : une sur les études relatives au vaccin antigrippal quadrivalent inactivé à virion fragmenté (Afluria MD Tetra, Seqirus) et une sur l'efficacité et l'immunogénicité comparatives des vaccins antigrippaux inactivés sous-unitaires et à virion fragmenté chez les adultes de 65 ans et plus. On peut trouver les détails complets dans la Déclaration sur la vaccination antigrippale pour la saison 2019-2020 (4) et les publications afférentes.

Le but de cet article est de résumer cette déclaration annuelle sur la grippe saisonnière.

\section{Mise à jour des abréviations relatives aux vaccins antigrippaux}

Les abréviations utilisées par le CCNI ont été mises à jour pour mieux décrire les caractéristiques des divers types de vaccins antigrippaux. Les nouvelles abréviations et leurs correspondances antérieures sont énumérées au tableau 1.

\section{Méthodologie}

Pour préparer la Déclaration sur la vaccination antigrippale pour la saison 2019-2020, le Groupe de travail sur l'influenza a ciblé deux revues de la littérature et, après la révision et l'analyse de l'information, a proposé des recommandations conformes aux processus basées sur des données probantes du CCNI (5). Le CCNI a évalué de manière critique les données probantes disponibles et a approuvé les recommandations particulières formulées.

\section{Utilisation du vaccin antigrippal Afluria Tetra}

Le Groupe de travail sur l'influenza a procédé à une revue systématique pour éclairer l'élaboration des directives du CCNI sur I'utilisation d'Afluria Tetra au Canada. Des recherches ont été effectuées dès le début dans cinq bases de données électroniques (MEDLINE, Embase, Scopus, ProQuest Public Health Database et ClinicalTrials.gov) jusqu'au 22 août 2017 pour trouver de la documentation pertinente sur l'efficacité potentielle, l'efficacité réelle, l'immunogénicité et l'innocuité d'Afluria Tetra ou d'Afluria trivalent (1,5\% de sodium taurodéoxycholate [TDOC]) chez les adultes et les enfants de

Tableau 1: Nouvelles abréviations du Comité consultatif national sur l'immunisations (CCNI) relatives aux vaccins contre l'influenza

\begin{tabular}{|c|c|c|c|c|}
\hline $\begin{array}{l}\text { Catégorie de vaccin } \\
\text { contre I'influenza }\end{array}$ & Formulation & Type & $\begin{array}{l}\text { Nouvelle abréviation } \\
\text { du CCNI }\end{array}$ & $\begin{array}{l}\text { Ancienne abréviation } \\
\text { du CCNI }\end{array}$ \\
\hline \multirow{7}{*}{$\begin{array}{l}\text { Vaccin inactivé contre } \\
\text { l'influenza }\end{array}$} & - & - & VII & VAl \\
\hline & \multirow{4}{*}{ Trivalent } & - & VII3 & VTI \\
\hline & & $\begin{array}{l}\text { À dose standard }{ }^{\mathrm{b}} \text {, sans adjuvant, administré par } \\
\text { voie IM }\end{array}$ & VII3-SD & VTI à dose normale \\
\hline & & Avec adjuvant ${ }^{c}$, administré par voie IM & VII3-Adj & VTla ou VTI avec adjuvant \\
\hline & & À haute dose ${ }^{d}$, sans adjuvant, administré par voie IM & VII3-HD & VTI à forte dose \\
\hline & \multirow[b]{2}{*}{ Quadrivalent } & - & VII4 & VAQ \\
\hline & & $\begin{array}{l}\text { À dose standard }{ }^{\mathrm{b}} \text {, sans adjuvant, administré par voie } \\
\text { IM }\end{array}$ & VII4-SD & VAQ à dose normale \\
\hline \multirow{3}{*}{$\begin{array}{l}\text { Vaccin vivant atténué contre } \\
\text { l'influenza }\end{array}$} & - & - & WAI & WAI \\
\hline & Trivalent & Vaporisateur nasal & WAI3 & VAAl trivalent \\
\hline & Quadrivalent & Vaporisateur nasal & WVAI4 & WAI quadrivalent \\
\hline \multicolumn{5}{|c|}{$\begin{array}{l}\text { Abréviations : IM, intramusculaire; VAI, vaccin antigrippal inactivé; } \\
\text { trivalent inactivé contre l'influenza; VII3-Adj, vaccin trivalent inactiv } \\
\text { contre l'influenza à dose standard; VIII, vaccin quadrivalent inactiv } \\
\text { l'influenza; VVAI3, vaccin trivalent vivant atténué contre l'influenza; } \\
\text { a Le suffixe numérique correspond au nombre d'antigènes conten } \\
\text { composé « -SD » renvoie aux produits VII sans adjuvant qui contier } \\
\text { un VII avec adjuvant (p. ex. VII3-Adj pour FluadMD ou Fluad Pédiatri } \\
\text { Haute dose) } \\
\text { b } 15 \mu \mathrm{g} \text { de HA par souche } \\
\text { c } 7,5 \mu \mathrm{g} \text { (dans } 0,25 \mathrm{~mL} \text { ) ou } 15 \mu \mathrm{g} \text { (dans } 0,5 \mathrm{mLI} \text { ) de HA par souche } \\
\text { d } 60 \mu \mathrm{g} \text { de HA par souche }\end{array}$} \\
\hline
\end{tabular}


six mois et plus. L'utilisation de $1,5 \%$ de TDOC comme agent de fractionnement a été intégrée au procédé de fabrication d'Afluria et du nouveau Afluria Tetra après qu'un signal d'innocuité pendant la saison grippale de 2010 en Australie dans I'hémisphère Sud a montré qu'Afluria nécessitant l'utilisation d'une quantité inférieure à 1,5\% de TDOC était associé à un taux accru de fièvre et de convulsions fébriles chez les enfants de moins de cinq ans (6). Deux évaluateurs ont examiné de manière indépendante les titres et les résumés d'articles recueillis dans le cadre de la recherche et les textes intégraux admissibles aux fins d'inclusion. Deux évaluateurs ont également saisi des données des études admissibles et déterminé la qualité méthodologique de ces études en utilisant les critères définis par Harris et coll. (7). Une synthèse narrative des données extraites a été réalisée.

\section{Efficacité et immunogénicité comparatives du vaccin antigrippal inactivé sous-unitaire et du vaccin antigrippal inactivé à virion fragmenté chez les adultes de 65 ans et plus}

Un examen rapide a été réalisé pour informer le CCNI sur les différences potentiellement importantes entre les vaccins antigrippaux inactivés sous-unitaires et les vaccins antigrippaux inactivés à virion fragmenté chez les adultes plus âgés. Des recherches ont été effectuées dans trois bases de données électroniques (MEDLINE, Embase, et ClinicalTrials. gov) pour trouver de la documentation pertinente publiée entre le $1^{\text {er }}$ janvier 2007 et le 13 octobre 2017 sur l'efficacité et l'immunogénicité des vaccins antigrippaux inactivés sous-unitaires et à virion fragmenté (sans adjuvant, dose standard) chez les adultes de 65 ans et plus. Une recherche manuelle des listes de référence des articles inclus a été réalisée en raison du nombre restreint de documents retrouvés dans la recherche initiale des bases de données. Un seul évaluateur a examiné les dossiers retrouvés et a procédé à l'extraction des données et à l'évaluation de la qualité des études admissibles. Une synthèse narrative des données extraites a été réalisée.

\section{Résultats}

\section{Utilisation du vaccin antigrippal Afluria Tetra}

En se basant sur l'analyse de deux essais contrôlés randomisés d'Afluria Tetra et deux d'Afluria (1,5\% TDOC) le CCNI a conclu qu'Afluria Tetra est sûr et ne présente pas une immunogénicité inférieure aux vaccins comparables chez les adultes et les enfants de cinq ans et plus. Aucune donnée probante directe quant à l'efficacité potentielle ou réelle n'est disponible. De plus, aucune donnée probante de tout résultat n'était disponible sur I'utilisation d'Afluria Tetra chez les enfants de moins de cinq ans et I'utilisation d'Afluria Tetra n'est pas autorisée pour ce groupe d'âge au Canada. La fièvre et les convulsions fébriles n'ont pas été définies comme préoccupations tant pour Afluria Tetra et
Afluria (1,5\% TDOC) dans deux études portant sur les enfants de cinq ans et plus.

Le CCNI recommande qu'Afluria Tetra puisse être considéré comme faisant partie des vaccins antigrippaux quadrivalents inactivés offerts aux adultes et enfants de cinq ans et plus (Recommandation discrétionnaire du CCNI, données probantes de catégorie B).

Des détails complets sur les conclusions de la revue de la littérature, l'analyse, les motifs et les considérations pertinentes à la recommandation se trouvent dans la Déclaration supplémentaire - Afluria Tetra (6).

Efficacité et immunogénicité comparatives du vaccin antigrippal inactivé sous-unitaire et du vaccin antigrippal inactivé à virion fragmenté chez les adultes de 65 ans et plus

On a identifié huit études qui ont évalué soit l'efficacité ou soit l'immunogénicité sous-unitaire du vaccin comparé aux vaccins antigrippaux inactivés à virion fragmenté. Ces études n'ont pas montré d'importantes différences statistiques dans l'efficacité ou l'immunogénicité du vaccin. De plus, la qualité des études retenues s'est avérée être préoccupante. En se basant sur un examen de ces études, le CCNI a conclu que les données probantes ne sont pas suffisantes (Données probantes de catégorie I) pour appuyer des recommandations précises sur l'utilisation différentielle des vaccins antigrippaux inactivés sous-unitaires et à virion fragmenté chez les adultes de 65 ans et plus.

Les conclusions complètes de cet examen se trouvent dans la Revue de la littérature du CCNI sur l'efficacité comparative et l'immunogénicité du vaccin antigrippal inactivé sous-unitaire et du vaccin antigrippal inactivé à virion fragmenté chez les adultes âgés de 65 ans et plus (8).

\section{Sommaire des recommandations du CCNI sur I'utilisation des vaccins antigrippaux pour la saison 2019-2020}

Le CCNI continue de recommander le vaccin antigrippal pour toute personne de six mois et plus qui ne présente aucune contre-indication à l'administration du vaccin. La vaccination devrait être offerte prioritairement aux personnes présentant un risque élevé de complications ou d’hospitalisation liées à la grippe, aux personnes capables de transmettre la grippe à des sujets à risque élevé et à d'autres personnes comme l'illustre le tableau 2.

Les options de vaccins antigrippaux recommandés par groupe d'âge et par dose et par voie d'administration par âge sont résumées au tableau 3 et au tableau 4 respectivement. 
Tableau 2 : Groupes pour lesquels le vaccin antigrippal est particulièrement recommandé

Personnes présentant un risque élevé de complications ou d'hospitalisation liées à la grippe:

- Toutes les femmes enceintes ${ }^{a}$

- Adultes et enfants atteints d'une des affections chroniques suivantes :

- $\quad$ maladies cardiaques ou pulmonaires (notamment dysplasie bronchopulmonaire, fibrose kystique et asthme)

- diabète sucré ou autres maladies métaboliques

- cancer, troubles liés à l'immunodépression (résultant d'une maladie sous-jacente, d'un traitement, ou des deux)

- néphropathie

- $\quad$ anémie ou hémoglobinopathie

- $\quad$ troubles neurologiques ou du développement neurologique (ces troubles comprennent les troubles neuromusculaires, neurovasculaires, neurodégénératifs et du développement neurologique ainsi que les troubles convulsifs [et, pour les enfants, les convulsions fébriles et les retards de développement isolés], mais ils excluent les migraines et les troubles psychiatriques sans troubles neurologiques)

- $\quad$ obésité morbide (indice de masse corporelle [IMC] au moins 40)

- $\quad$ enfants et adolescents (de 6 mois à 18 ans) sous traitement pendant de longues périodes par de l'acide acétylsalicylique, en raison de la possibilité d'un risque accru de syndrome de Reye associé à la grippe

- Résidents de maisons de soins infirmiers et d'autres établissements de soins de longue durée, quel que soit leur âge

- Personnes de 65 ans et plus

- Tous les enfants de 6 à 59 mois

- Autochtones

Personnes qui pourraient transmettre la grippe à des sujets à risque élevé:

- Travailleurs de la santé (TS) et autres fournisseurs de soins dans des établissements et en milieux communautaires qui, par leurs activités, pourraient transmettre la grippe à des sujets à risque élevé de complications

- Contacts familiaux (adultes et enfants) de personnes à risque élevé de complications liées à la grippe, que ces dernières aient été vaccinées ou non :

- contacts familiaux de personnes à risque élevé

- contacts familiaux des nourrissons âgés de moins de 6 mois, qui sont à risque élevé de complications grippales, mais qui ne peuvent pas recevoir un vaccin contre la grippe

- membres d'un ménage devant accueillir un nouveau-né durant la saison grippale

- Personnes qui s'occupent régulièrement d'enfants âgés de 6 à 59 mois, que ce soit à la maison ou à l'extérieur

- Personnes qui fournissent des services à des sujets à risque élevé dans un milieu fermé ou relativement fermé (p. ex. équipage de navire)

Autres:

- Personnes qui fournissent des services communautaires essentiels

- Personnes en contact direct avec de la volaille infectée par le virus de la grippe aviaire durant les activités d'abattage

a Le risque d'hospitalisation liée à la grippe augmente avec la durée de gestation, c'est-à-dire qu'il est plus élevé pendant le troisième trimestre que pendant le deuxième 
Tableau 3: Recommandations concernant le choix du vaccin antigrippal pour le processus décisionnel à l'échelle individuellea ${ }^{a}$ par groupe d'âge (4)

\begin{tabular}{|c|c|c|}
\hline Groupe d'âge & $\begin{array}{l}\text { Types de } \\
\text { vaccins } \\
\text { disponibles }\end{array}$ & Recommandations concernant le choix du vaccin antigrippal \\
\hline $\begin{array}{l}\text { Enfants de } \\
6 \text { à } 23 \text { mois }\end{array}$ & $\begin{array}{ll}\text { - } & \text { VII3-SD } \\
\text { - } & \text { VII3-Adj } \\
\text { VIII4-SD }\end{array}$ & $\begin{array}{l}\text { - Le vaccin quadrivalent contre la grippe doit être utilisé, compte tenu du fardeau du virus de } \\
\text { la grippe B dans ce groupe d'âge et de la possibilité de non-concordance entre la souche } \\
\text { prédominante du virus de la grippe B en circulation et la souche d'un vaccin trivalent } \\
\text { - En l'absence d'un vaccin quadrivalent, l'un ou l'autre des vaccins trivalents doit être utilisé }\end{array}$ \\
\hline $\begin{array}{l}\text { Enfants et adolescents } \\
\text { de } 2 \text { à } 17 \text { ans }\end{array}$ & $\begin{array}{ll}- & \text { VII3-SD } \\
\text { - } & \text { VII4-SD } \\
\text { - VVAI4 }\end{array}$ & $\begin{array}{l}\text { - Le VII4-SD ou le VVAI4 doit être utilisé pour les enfants et adolescents qui ne présentent pas } \\
\text { de contre-indications, y compris ceux atteints d'une affection chronique n'entraînant pas de } \\
\text { déficit immunitaire, compte tenu du fardeau du virus de la grippe B dans ce groupe d'âge et } \\
\text { de la possibilité de non-concordance entre la souche prédominante du virus de la grippe B en } \\
\text { circulation et la souche d'un vaccin trivalent } \\
\text { - } \quad \text { En l'absence d'un VII4-SD ou VVAI4, il faut utiliser le VII3-SD } \\
\text { - Le VII4-SD doit être administré aux enfants et adolescents pour qui le VVAl est contre-indiqué, par } \\
\text { exemple : } \\
\text { - les sujets atteints d'asthme grave } \\
\text { - les sujets ayant une respiration sifflante qui a nécessité une intervention médicale au } \\
\text { - leurs des sept jours précédant la vaccination } \\
\text { - l'Aspirine } \\
\text { - les sujets immunodéprimés } \\
\text { - } \quad \text { aux sujets atteints d'une forme d'asthme non grave et stable } \\
\text { - aux sujets atteints de fibrose kystique qui ne sont pas traités par des médicaments } \\
\text { immunosuppresseurs, tels des corticostéroïdes à action générale à long terme }\end{array}$ \\
\hline $\begin{array}{l}\text { Adultes de } \\
18 \text { à } 59 \text { ans }\end{array}$ & $\begin{array}{ll}- & \text { VII3-SD } \\
\text { - } & \text { VII4-SD } \\
\text { - VVAI4 }\end{array}$ & $\begin{array}{l}\text { - L'un ou l'autre des vaccins antigrippaux disponibles peut être utilisé chez les adultes qui ne } \\
\text { présentent aucune contre-indication } \\
\text { - Le VII doit être administré aux adultes pour qui le VVAl est contre-indiqué, par exemple : } \\
\text { - les femmes enceintes } \\
\text { - les adultes atteints d'une affection chronique figurant dans le tableau 2, y compris un } \\
\text { - } \quad \text { déficit } \\
\text { - limmunitaire }\end{array}$ \\
\hline $\begin{array}{l}\text { Adultes de } \\
60 \text { à } 64 \text { ans }\end{array}$ & $\begin{array}{ll}\text { - } & \text { VII3-SD } \\
\text { - } & \text { VII4-SD }\end{array}$ & - L'un ou l'autre des vaccins antigrippaux disponibles peut être utilisé \\
\hline $\begin{array}{l}\text { Adultes de } 65 \text { ans et } \\
\text { plus }^{\mathrm{b}}\end{array}$ & $\begin{array}{ll}\text { - } & \text { VII3-SD } \\
\text { - } & \text { VII3-Adj } \\
\text { - } & \text { VII3-HD } \\
\text { - VII4-SD }\end{array}$ & $\begin{array}{l}\text { - S'il est offert, le VII3-HD devrait être utilisé plutôt que le VII3-SD, compte tenu du fardeau de } \\
\text { la maladie associée à la grippe A(H3N2) et des données probantes suffisantes à l'appui d'une } \\
\text { efficacité supérieure du VII3-HD par rapport au VII3-SD dans ce groupe d'âge } \\
\text { - Les données probantes sont insuffisantes pour formuler des recommandations comparatives à } \\
\text { l'échelle individuelle sur l'utilisation du VII3-Adj ou du VII4-SD plutôt que du VII3-SD ou entre le } \\
\text { VII3-Adj, le VII3-HD et le VII4-SD }\end{array}$ \\
\hline
\end{tabular}

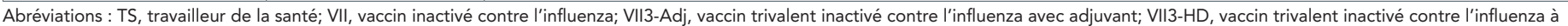

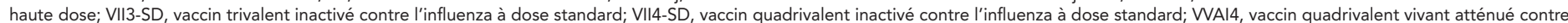

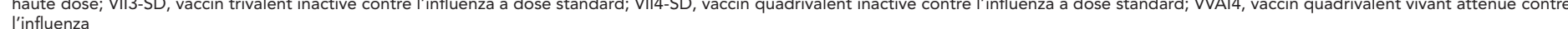

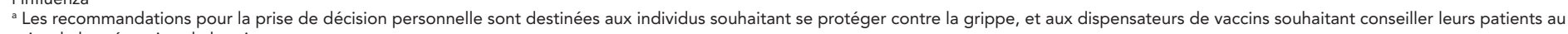
sujet de la prévention de la grippe

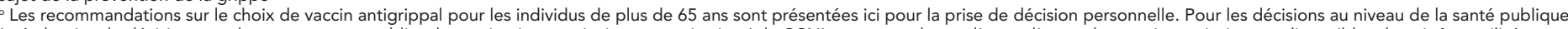

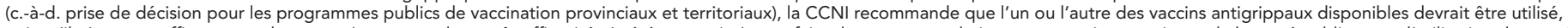

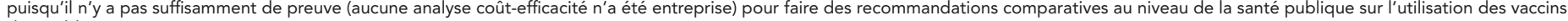
disponibles 
Tableau 4: Posologie et voie d'administration recommandées, selon l'âge, pour les types de vaccins antigrippaux offerts pour la saison 2019-2020 (4)

\begin{tabular}{|c|c|c|c|c|c|}
\hline \multirow{2}{*}{ Groupe d'âge } & \multicolumn{4}{|c|}{$\begin{array}{l}\text { Type de vaccin antigrippal } \\
\text { (voie d'administration) }\end{array}$} & \multirow{2}{*}{$\begin{array}{c}\text { Nombre de } \\
\text { doses requises }\end{array}$} \\
\hline & $\begin{array}{c}\text { VII3-SD }{ }^{\mathrm{a}} \text { ou } \\
\text { VII4-SD } \\
\text { (intramusculaire) }\end{array}$ & $\begin{array}{c}\text { VII3-Adj }^{c} \\
\text { (intramusculaire) }\end{array}$ & $\begin{array}{c}\text { VII3-HDd } \\
\text { (intramusculaire) }\end{array}$ & $\begin{array}{c}\mathrm{VVAl}^{\mathrm{e}} \\
\text { (intranasal) }\end{array}$ & \\
\hline 6 à 23 mois & $0,5 \mathrm{~mL}^{f}$ & $0,25 \mathrm{~mL}$ & - & - & 1 ou $2^{g}$ \\
\hline 2 à 8 ans & $0,5 \mathrm{~mL}$ & - & - & $\begin{array}{c}0,2 \mathrm{~mL} \\
(0,1 \mathrm{~mL} \text { par narine })\end{array}$ & 1 or $2^{g}$ \\
\hline 9 à 17 ans & $0,5 \mathrm{~mL}$ & - & - & $\begin{array}{c}0,2 \mathrm{~mL} \\
(0,1 \mathrm{~mL} \text { par narine })\end{array}$ & 1 \\
\hline 18 à 59 ans & $0,5 \mathrm{~mL}$ & - & - & $\begin{array}{c}0,2 \mathrm{~mL} \\
(0,1 \mathrm{~mL} \text { par narine })\end{array}$ & 1 \\
\hline 60 à 64 ans & $0,5 \mathrm{~mL}$ & - & - & - & 1 \\
\hline 65 ans et plus & $0,5 \mathrm{~mL}$ & $0,5 \mathrm{~mL}$ & $0,5 \mathrm{~mL}$ & - & 1 \\
\hline \multicolumn{6}{|c|}{ 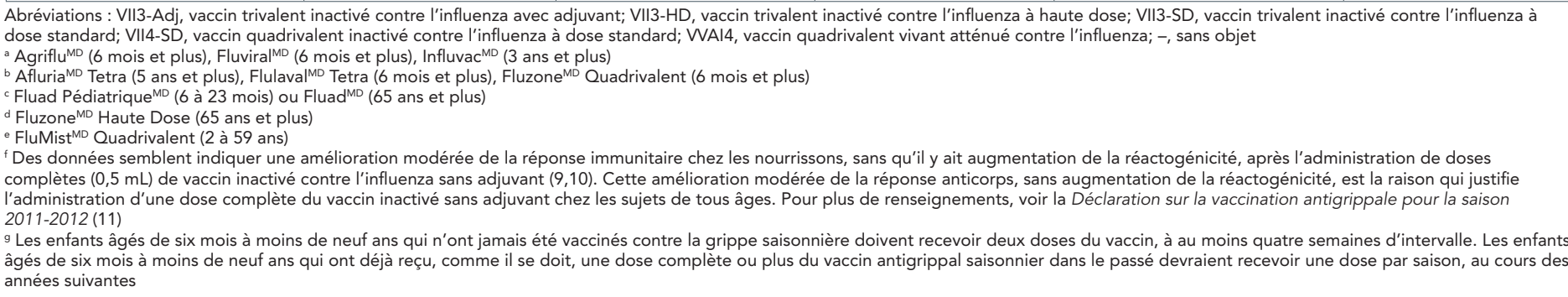 } \\
\hline
\end{tabular}

\section{Conclusion}

Le CCNI continue de recommander la vaccination antigrippale annuelle de toutes les personnes de six mois et plus (tenir compte des indications et des contre-indications particulières à l'âge des produits particuliers) en accordant une importance spéciale aux personnes présentant un risque élevé de complications ou d'hospitalisation liées à la grippe. Cela inclut les femmes enceintes, les personnes pouvant transmettre la grippe à des sujets à risque élevé, les personnes qui fournissent des services communautaires essentiels et les personnes en contact direct avec de la volaille infectée par le virus de la grippe aviaire durant les activités d'abattage. Pour la saison grippale 2019-2020, le CCNI recommande que ce nouveau vaccin Afluria Tetra puisse compter parmi les vaccins antigrippaux quadrivalents inactivés offerts aux adultes et aux enfants de cinq ans et plus. Le CCNI a cependant conclu que les données probantes ne sont pas suffisantes en ce moment pour appuyer des recommandations précises sur l'utilisation différentielle des vaccins antigrippaux inactivés sous-unitaire et à virion fragmenté chez les adultes de 65 ans et plus.

\section{Déclaration des auteurs}

L. Z. - Rédaction - rédaction de la première ébauche - révision

K. Y. - Rédaction - rédaction de la première ébauche - révision

I. G. - Rédaction - rédaction de la première ébauche - révision
Le Chapitre sur la grippe du Guide canadien d'immunisation et la Déclaration sur la vaccination antigrippale pour la saison 2019-2020 a été préparé par L. Zhao, K. Young, R. Stirling et I. Gemmill, et a été approuvé par le CCNI.

\section{Conflit d'intérêts}

Aucun.

\section{Remerciements}

Membres du groupe de travail sur la grippe : I. Gemmill (Président), C. Bancej, L. Cochrane, N. Dayneka, L. Grohskopf, D. Kumar, J. Langley, M. Lavoie, P. Lebans, J. McElhaney, A. McGeer, D. Moore, B. Warshawsky et J. Xiong

Membres du CCNI : C. Quach (Présidente), W. Vaudry (Vice-présidente), N. Dayneka, P. De Wals, S. Deeks, V. Dubey, R. Harrison, M. Lavoie, C. Rotstein, M. Salvadori, B. Sander, N. Sicard et R. Warrington

Agents de liaison : J. Brophy (Association canadienne pour la recherche et l'évaluation en immunisation), E. Castillo (Société des obstétriciens et gynécologues du Canada), A. Cohn (Centers for Disease Control and Prevention, États-Unis), T. Cole (Comité canadien pour l'immunisation), J. Emili (le Collège des médecins de famille du Canada), K. Klein (Conseil des médecins hygiénistes 
en chef), C. Mah (Association canadienne de santé publique), D. Moore (Société canadienne de pédiatrie) et A. Pham-Huy (Association pour la microbiologie médicale et l'infectiologie Canada)

Représentants d'office : K. Barnes (Défense nationale et Forces armées canadiennes), E. Henry (Centre de l'immunisation et des maladies respiratoires infectieuses [CIMRI], Agence de la santé publique du Canada [ASPC]), G. Coleman (Direction des produits biologiques et des thérapies génétiques, Santé Canada [SC]), J. Gallivan (Direction des produits de santé commercialisés, SC), J. Pennock (CIMRI, ASPC), G. Poliquin (Laboratoire national de microbiologie, ASPC) et T. Wong (Direction générale de la Santé des Premières nations et des Inuits, Services aux Autochtones Canada)

Le CCNI reconnaît la contribution de M. Doll, A. Fleurant, A. House, M. Laplante et $M$. Tunis à cette Déclaration et leur en est reconnaissant.

\section{Aide financière}

Les travaux du Comité consultatif national de l'immunisation sont soutenus par l'Agence de la santé publique du Canada.

\section{Références}

1. Statistique Canada. Les dix principales causes de décès, 2011. Ottawa (ON): Statistique Canada; 2014 (Accédé 2019-04-16). https://www150.statcan.gc.ca/n1/pub/82625-x/2014001/article/11896-fra.htm

2. Schanzer DL, McGeer A, Morris K. Statistical estimates of respiratory admissions attributable to seasonal and pandemic influenza for Canada. Influenza Other Respir Viruses 2013 Sep;7(5):799-808. DOI PubMed

3. Schanzer DL, Sevenhuysen C, Winchester B, Mersereau T. Estimating influenza deaths in Canada, 1992-2009. PLoS One 2013 Nov;8(11):e80481. DOI PubMed

4. Comité consultatif national de l'immunisation (CCNI). Chapitre sur la grippe du Guide canadien d'immunisation et Déclaration sur la vaccination antigrippale pour la saison 2019-2020. Ottawa (ON) : Agence de la santé publique du Canada; 2019. https://www.canada.ca/fr/ sante-publique/services/publications/vaccins-immunisation/ guide-canadien-immunisation-declaration-vaccinationantigrippale-2019-2020.html
5. Comité consultatif national de l'immunisation (CCNI). Recommandations pour l'immunisation fondées sur des données probantes - Méthodes du Comité consultatif national de l'immunisation. Relevé des maladies transmissibles au Canada 2009 Jan;35(DCC-1):1-10. https://www.canada.ca/fr/sante-publique/services/ rapports-publications/releve-maladies-transmissibles-canadarmtc/numero-mensuel/2009-35/methodes-comit e-consultatif-national-immunisation.html

6. Comité consultatif national de l'immunisation (CCNI). Déclaration supplémentaire - Afluria Tetra ${ }^{\mathrm{MD}}$. Ottawa (ON): I'Agence de la santé publique du Canada; 2019. (Accédé 2019-01-31) https://www.canada.ca/fr/sante-publique/ services/publications/vie-saine/declaration-supplementair e-afluria-tetra.html

7. Harris RP, Helfand $M$, Woolf $\mathrm{SH}$, Lohr KN, Mulrow CD, Teutsch SM, Atkins D; Methods Work Group, Third US Preventive Services Task Force. Current methods of the US Preventive Services Task Force: a review of the process. Am J Prev Med 2001 Apr;20(3 Suppl):21-35. DOI PubMed

8. Comité consultatif national de l'immunisation (CCNI). Revue de la littérature du CCNI sur l'efficacité comparative et l'immunogénicité du vaccin antigrippal inactivé sous-unitaire et du vaccin antigrippal inactivé à virion fragmenté chez les adultes âgés de 65 ans et plus. Ottawa (ON): l'Agence de la santé publique du Canada; 2018. https:// www.canada.ca/fr/sante-publique/services/publications/ vie-saine/revue-litterature-efficacite-comparative-immunog enicite-vaccin-antigrippal-inactive-sous-unitaire-vacci n-antigrippal-inactive-virion-fragmente-chez-adultes-ages65-ans-plus.html

9. Langley JM, Vanderkooi OG, Garfield HA, Hebert J, Chandrasekaran V, Jain VK, Fries L. Immunogenicity and safety of 2 dose levels of a thimerosal-free trivalent seasonal influenza vaccine in children aged 6-35 months: a randomized, controlled trial. J Pediatric Infect Dis Soc 2012 Mar;1(1):55-63. DOI PubMed

10. Skowronski DM, Hottes TS, Chong M, De Serres G, Scheifele DW, Ward BJ, Halperin SA, Janjua NZ, Chan T, Sabaiduc S, Petric M. Randomized controlled trial of dose response to influenza vaccine in children aged 6 to 23 months. Pediatrics 2011 Aug;128(2):e276-89. DOI PubMed

11. Comité consultatif national de l'immunisation (CCNI). Déclaration sur la vaccination antigrippale pour la saison 2011-2012. Relevé des maladies transmissibles au Canada 2011;37(DCC-5):1-61. DOI 